\title{
A Novel Multi-minislot Cooperative Spectrum Sensing Scheme in Cognitive Radio Networks
}

\author{
Hemendra Dhurvey \\ Department of ECE \\ MANIT, Bhopal \\ India
}

\author{
Hirdesh Chack \\ Department of ECE \\ MANIT, Bhopal \\ India
}

\author{
O.P. Meena \\ Department of ECE \\ MANIT, Bhopal \\ India
}

\begin{abstract}
The use of Cognitive Radio Network (CRN) for spectrum utilization is very beneficial to address scarcity of frequency spectrum. It can solve the problem of spectrum scarcity. The one of the areas of concern in spectrum sensing is the amount of energy that is consumed in spectrum sensing performed by unlicensed/secondary users. There are many frame structures which are being used for spectrum sensing and they are very capable of performing spectrum efficient and energy efficient spectrum sensing individually, but it does not fulfill the need of modern era where we need a combined spectrum and energy efficient spectrum sensing technique. The proposed time division based frame structure for multiuser CRN is spectrum and energy efficient, it also provides time diversity gain for secondary users by allowing collection of sensing results at different point of time. The simulation result shows that the spectrum utility also increases because of the use of optimal decision threshold for final decision at fusion center. The results also shows that all the secondary users can't be used for spectrum sensing because it degrades energy efficiency. So it is also optimized that how many secondary users will perform energy efficient spectrum sensing.
\end{abstract}

\section{General terms}

Spectrum utilization, Cognitive radio networks

\section{Keywords}

Utility maximization, multi-minislot, spectrum sensing, cooperative spectrum sensing, Utility function

\section{INTRODUCTION}

The increased popularity and utilization of wireless communication has created scarcity of frequency spectrum. The static spectrum allocation policy by government agencies has lead to underutilization of spectrum utilization. A study by FCC (Federal Communication Commission) shows that the spectrum utilization varies from $15 \%$ to $85 \%$ [1] which is function of time and geography.

The CRN is the technology which allows opportunistic utilization of unused licensed spectrum to the unlicensed/ secondary users in absence of signal of licensed/primary users. The secondary users (SU) have capability to sense the spectrum [2]. There are different types of frame structures for spectrum sensing and SU's data transmission in the CRN. The basic and conventional frame structures are based on frequency and time division techniques but energy consumption is very high by the cognitive radio devices $[3,4]$. We need to design a frame structures which is both energy and spectrum efficient. The proposed frame structures in this allows energy efficient spectrum sensing in multi user Cooperative Spectrum Sensing (CSS) in CRN

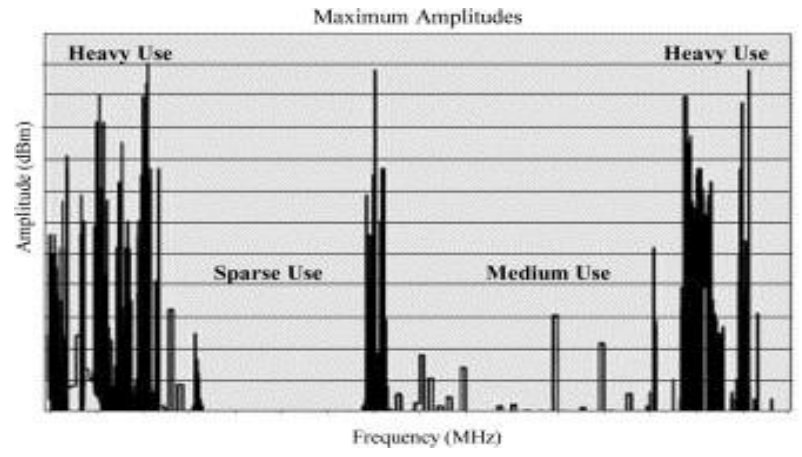

Fig.1.Spectrum utilization

\section{SPECTRUM SENSING FRAME WORKS}

\subsection{Single user time division spectrum sensing framework (TDSSF)}

In CRN different types of time division and frequency division based spectrum sensing frameworks are used. In Time Division Spectrum Sensing Framework (TDSSF) as shown in Fig. 2 [5], in sensing time slot the SU senses the spectrum while in data transmission time slot it transmits its own data if the primary signal detected absent over the complete primary spectrum.

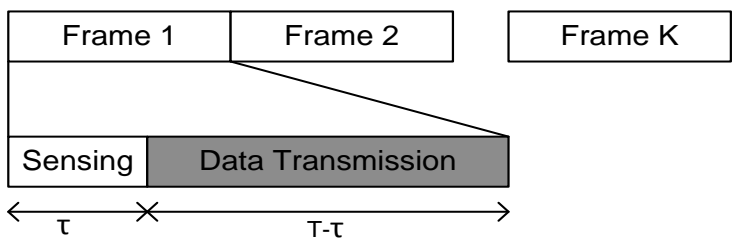

Fig.2 Single user time division spectrum sensing framework

In the frame the frame duration is denoted as T, sensing time is denoted as $t_{s}$, and data transmission time is denoted as T- $t_{s}$. Using TDSSF the SU senses the spectrum periodically in every frame. The periodic spectrum sensing and leads to regular interruption in SU's data transmission in absence of primary signal.

\subsection{Single user frequency division spectrum sensing framework (FDSSF)}

The continuous sensing of the PU's spectrum improves spectrum detection probability. Therefore, to alleviate the SU interruption during its data transmission using TDSSF and to improve spectrum detection probability, the PU frequency band is divided into two sub bands, one for opportunistic SU data transmission, and the other for continuous spectrum sensing [6] as shown in Fig. 3. The average SU transmission delay is reduced by selecting the proper bandwidth for spectrum 
sensing within each frame. Since different SUs may have different requirements on their quality of services, so the achievable average SU throughput is maximized by choosing the optimal sensing bandwidth within multiple adjacent frames.

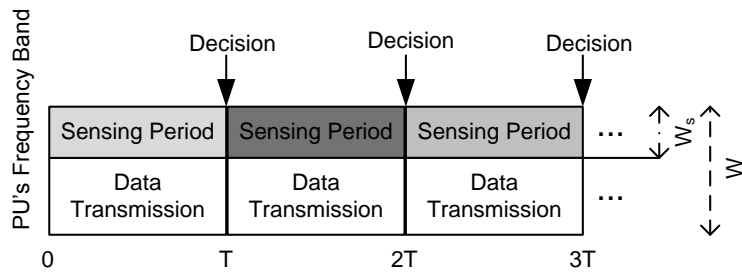

Fig.3. Single user Frequency division spectrum sensing framework

\subsection{Conventional multi-user cooperative spectrum sensing system model}

The Fig.4 shows the conventional frame structure used in cooperative spectrum sensing there are three blocks, sensing block, reporting block, and data transmission block. The frame

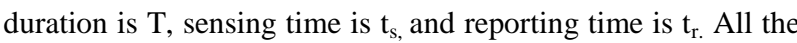
secondary users sense in the sensing block and send their results to fusion center one after one. If fusion center takes decision that the primary user is present they initiate data transmission otherwise they start sensing again $[7,8,9]$.

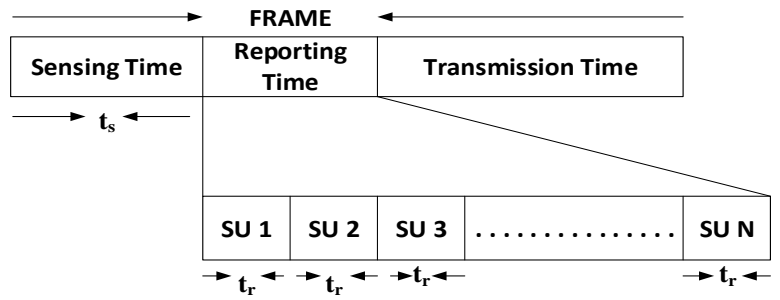

Fig, 4 conventional cooperative sensing system model

\subsection{Proposed multi-user cooperative spectrum sensing system model}

In proposed cooperative spectrum sensing frame structure the reporting time of one secondary user is used as the sensing time for other secondary user it helps in improving spectrum efficiency, it was not the case with conventional frame structure based spectrum sensing.

The frame structure of new cooperative sensing frame structure is shown in the Fig.5 [10]. The focus in this frame structure is to increase the sensing time for achieving greater spectrum efficiency without additional time overhead and this can be done by utilizing the reporting block for sensing purpose. To do this secondary users have to sense and report to the fusion center at the same and use reporting time of one secondary for sensing of all other secondary users . By doing this we have $(\mathrm{N}-1) \mathrm{t}_{\mathrm{r}}$ reporting time in $\mathrm{m}$ frame and $\mathrm{t}_{\mathrm{s}}$ reporting time in $\mathrm{m}+1$ frame for performing spectrum sensing this results in greater spectrum efficiency because sensing results are sampled for long time.

The energy detection and decision fusion are used in cooperative spectrum sensing to optimize utility function. In cognitive spectrum sensing using decision fusion all secondary users take their own decision and send single bit decision to the fusion Center. The fusion Center collects all single bit decisions and uses them to take the final decision.The final decision is also depends on the fusion rule used.

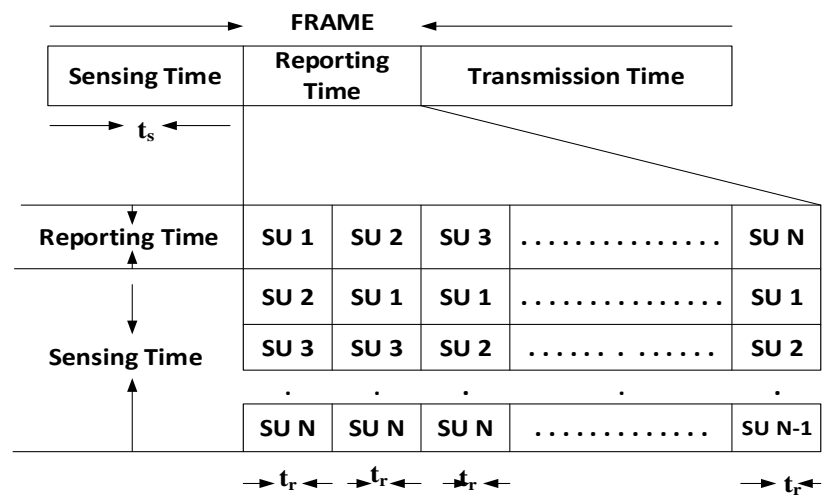

Fig. 5 New frame structure for CSS

\section{MATHEMATICAL ANALYSIS}

\subsection{Single user spectrum sensing}

The signal $V_{i}(\mathrm{n})$ received by $i$ th secondary user can be formulated as given in eq. (1) [11]. The presence and absence of primary signal is distinguished by differentiating the hypothesis $\mathrm{H}_{0}$ (primary user is absent) and $\mathrm{H}_{1}$ (primary user is present)

$V_{i}(\mathrm{n})=\left\{\begin{array}{c}\varepsilon_{\mathrm{i}(\mathrm{n}),} \\ \mathrm{h}_{\mathrm{i}} \mathrm{s}(\mathrm{n})+\varepsilon_{\mathrm{i}(\mathrm{n}),}\end{array}\right.$

Where $\mathrm{n}=1,2, \ldots \ldots, \mathrm{u}, \mathrm{u}$ is number of samples collected by secondary users of received signal while sensing. It is the product of sensing time $t_{s}$ and sampling frequency $\mathrm{f}_{\mathrm{s}}$. And $\mathrm{s}(\mathrm{n})$ is the primary user signal, $\varepsilon_{\mathrm{i}(\mathrm{n})}$ is noise and identically distributed and assumed to be independent, real valued Gaussian variable zero mean variance $\mathrm{E}\left[|\varepsilon(i)(n)|^{2}\right]=\sigma^{2} \cdot \mathrm{h}_{\mathrm{i}}$ is channel gain .

Then SNR of primary user at the $i$ th secondary user can be expressed as

$\gamma_{i}=\frac{\left|h_{i}\right|^{2} \mathrm{E}\left[|s(n)|^{2}\right]}{\sigma^{2}}$

The decision statistic for energy detection is $V_{i}=\frac{1}{u} \sum_{n=1}^{u}\left|v_{i}(n)\right|^{2}$. the sub script $\mathrm{i}$ can be omitted because primary user signal is considered to be i.i.d [12]. for large $t_{s} \mathrm{f}_{\mathrm{s}}$ using central limit theorem, we have

$V \sim\left\{\begin{array}{cc}\mathrm{N}\left(\sigma^{2}, \frac{2 \sigma^{4}}{t_{s} f_{s}}\right), & H_{0} \\ \mathrm{~N}\left((\gamma+1) \sigma^{2}, \frac{2(2 \gamma+1) \sigma^{4}}{t_{s} f_{s}}\right), & H_{1}\end{array}\right.$

Then probability density function can be written as

$f_{V}(v)=\frac{\sqrt{t_{s} f_{s}}}{2 \sigma^{2} \sqrt{\pi}} e^{-\frac{t_{S} f_{S}\left(v-\sigma^{2}\right)^{2}}{4 \sigma^{4}}}, \quad H_{0}$

$f_{\mathrm{V}}(v)=\frac{\sqrt{t_{s} \mathrm{f}_{s}}}{2 \sigma^{2} \sqrt{\pi(2 \gamma+1)}} \mathrm{e}^{-\frac{t_{s} \mathrm{f}_{\mathrm{s}}\left[v-(\gamma+1) \sigma^{2}\right]^{2}}{4(2 \gamma+1) \sigma^{4}}}, \quad \mathrm{H}_{1}$

Then the probability of detection and probability of false alarm can be determined by [13]. 


$$
\begin{aligned}
& p_{f}=\mathrm{P}\left\{V>\lambda \mid H_{0}\right\}=\int_{\lambda}^{\infty} f_{V \mid H_{0}}(v) d v=Q\left(\left(\frac{\lambda}{\sigma^{2}}-1\right) \sqrt{\frac{t_{\frac{s}{2} \mathbf{f}_{s}}}{2}}\right) \\
& p_{d}=\mathrm{P}\left\{V>\lambda \mid H_{1}\right\}=\int_{\lambda}^{\infty} f_{V \mid H_{1}}(v) d v=Q\left(\left(\frac{\lambda}{\sigma^{2}}-\gamma-1\right) \sqrt{\frac{t_{s} \mathbf{f}_{s}}{2(2 \gamma+1)}}\right)
\end{aligned}
$$

Where $\lambda$ is energy detection threshold, $\mathrm{Q}($.$) is the function$ defined as $Q(x)=\frac{1}{\sqrt{2 \pi}} \int_{x}^{\infty} e^{-\frac{t^{2}}{2}} d t$. The probability of false alarm should be as low as possible and probability of detection should be as high as possible for greater spectrum efficiency. The energy consumed by secondary users should be low for energy efficiency. Considering all three factors the economical utility function is defined as [10].

$U_{1}=\mathrm{p}\left(H_{0}\right)\left(1-\mathrm{p}_{\mathrm{f}}\right) \frac{T-t_{s}}{T} \phi_{1}-\mathrm{p}\left(H_{1}\right)\left(1-\mathrm{p}_{\mathrm{d}}\right) \frac{T-t_{s}}{T} \phi_{2}-\frac{t_{s}}{T} \phi_{3}$

Where $\mathrm{p}\left(\mathrm{H}_{0}\right)$ is the probability that primary user is absent and $\mathrm{p}\left(\mathrm{H}_{1}\right)$ is the probability that primary user is present, the first part is related to spectrum efficiency, the second part is related to the disadvantage of missed detection due to the interference caused by secondary user to primary user, and the third and last part is related to the cost of energy consumed.

The maximum utility can be achieved by optimizing the threshold $\lambda$. The optimal threshold can be given by [14]

$\lambda_{\text {opt }}=\arg _{\lambda} \max U_{1}$

When $\frac{\partial U_{1}}{\partial \lambda}=0$.

By deriving expression for $\frac{\partial U_{1}}{\partial \lambda}$, we have

$\frac{\partial U_{1}}{\partial \lambda}=-\mathrm{p}\left(H_{0}\right) \frac{T-t_{s}}{T} \phi_{1} \cdot \frac{\mathrm{dp}_{\mathrm{f}}}{\mathrm{d} \lambda}+\mathrm{p}\left(\mathrm{H}_{1}\right) \frac{T-t_{s}}{T} \phi_{2} \cdot \frac{\mathrm{dp}_{\mathrm{d}}}{\mathrm{d} \lambda}$

Where

$$
\begin{aligned}
\frac{\mathrm{dp}_{\mathrm{f}}}{\mathrm{d} \lambda} & =-\frac{1}{2 \sigma^{2}} \sqrt{\frac{t_{s} f_{s}}{\pi}} e^{-\frac{t_{s} f_{s}}{4}}\left(\frac{\lambda}{\sigma^{2}}-1\right)^{2} \\
\frac{\mathrm{dp}}{\mathrm{d} \lambda} & =-\frac{1}{2 \sigma^{2}} \sqrt{\frac{t_{s} f_{s}}{\pi(2 \gamma+1)}} e^{-\frac{t_{s} f_{s}}{4(2 \gamma+1)}}\left(\frac{\lambda}{\sigma^{2}}-\gamma-1\right)^{2}
\end{aligned}
$$

Then optimal threshold can be calculated as

$\lambda_{\mathrm{opt}}=\frac{\sigma^{2}}{2}\left(1+\sqrt{1+2 \gamma+\frac{8(2 \gamma+1)}{\gamma t_{s} f_{s}}} \ln \frac{\sqrt{2 \gamma+1} \mathrm{p}\left(H_{0}\right) \phi_{1}}{\mathrm{p}\left(H_{1}\right) \phi_{2}}\right)$.

\subsection{Multi-minislot cooperative spectrum sensing}

Let $\Phi$ be the number of secondary users reporting the presences of primary user. Then the final decision can be expressed as

If $\Phi>\mathrm{k}$ decide $\mathrm{H}_{1}$

If $\Phi=\mathrm{k}$ decide $\mathrm{H}_{1}$ with probability $\alpha(0 \leq \alpha \geq 1)$,

If $\Phi<\mathrm{k}$ decide $\mathrm{H}_{0}$,

Where $\mathrm{k}$ is an integer, and $\mathrm{k}=1,2, \ldots \ldots \ldots \ldots \ldots, \mathrm{N}$ is decision threshold at the fusion Center. We need to optimize $\mathrm{k}$ in such a manner that it maximizes the utility function that to without introducing additional overhead $[15,16]$.

The probability of false alarm and the probability of detection for perfect reporting channel can be given by

$Q_{f}=\sum_{i=k+1}^{N}\left(\begin{array}{l}N \\ i\end{array}\right) \mathrm{p}_{\mathrm{f}}^{i}\left(1-\mathrm{p}_{\mathrm{f}}\right)^{N-i}+\alpha\left(\begin{array}{l}N \\ k\end{array}\right) \mathrm{p}_{\mathrm{f}}^{k}\left(1-\mathrm{p}_{\mathrm{f}}\right)^{N-k}$,
$Q_{d}=\sum_{i=k+1}^{N}\left(\begin{array}{l}N \\ i\end{array}\right) \mathrm{p}_{\mathrm{d}}^{i}\left(1-\mathrm{p}_{\mathrm{d}}\right)^{N-i}+\alpha\left(\begin{array}{l}N \\ k\end{array}\right) \mathrm{p}_{\mathrm{d}}^{k}\left(1-\mathrm{p}_{\mathrm{d}}\right)^{N-k}$

When $\mathrm{k}=\mathrm{N}$ first part of both the equation becomes zero

In reality it is difficult to have perfect reporting channels there may be error in the bits send by secondary users to the fusion center so, it should be detected at the fusion center before taking the final decision

Let $\mathrm{L}$ is the local decision bit, and $\mathrm{D}$ is the received bit by fusion center, we have

$\mathrm{P}\{\mathrm{D}=1 \mid \mathrm{L}=0\}=\mathrm{P}\{\mathrm{D}=0 \mid L=1\}=\mathrm{p}_{e}$,

$\mathrm{P}\{\mathrm{D}=1 \mid \mathrm{L}=1\}=\mathrm{P}\{\mathrm{D}=0 \mid L=0\}=1-\mathrm{p}_{e} ，$

The false alarm and detection probabilities of each SU for imperfect reporting channels will be given by

$\overline{\mathrm{P}}_{\mathrm{f}}=\left(1-2 \mathrm{p}_{\mathrm{e}}\right) \cdot \mathrm{p}_{\mathrm{f}}+\mathrm{p}_{\mathrm{e}}$ ，

$\overline{\mathrm{P}_{\mathrm{d}}}=\left(1-2 \mathrm{p}_{\mathrm{e}}\right) \cdot \mathrm{p}_{\mathrm{d}}+\mathrm{p}_{\mathrm{e}}$ ，

Where $\mathrm{p}_{\mathrm{e}}$ is reporting error if reporting error is equal to zero the equation (18) and (19) will be equal to (6) and (7) respectively

The secondary users are far away from fusion Center they need energy to send their decision to fusion center .considering the optimization of cooperative spectrum sensing using energy detection to maximize the utility function[1]. The Utility function can be defined as [10]

$U_{2}=\mathrm{p}\left(H_{0}\right)\left(1-Q_{f}\right) \frac{T-t_{s}-N t_{r}}{T} \phi_{1}-\mathrm{p}\left(H_{1}\right)\left(1-Q_{d}\right) \frac{T-t_{s}-N t_{r}}{T} \phi_{2}$

$-\frac{N t_{s}}{T} \phi_{3}-\frac{N t_{r}}{T} \phi_{4}$

Where first part represents spectrum efficiency, the second part represents the disadvantage of missed detection, the third part represents the cost of energy used for sensing, and the fourth part represents the cost of energy used for reporting to fusion center. $\phi_{1}, \phi_{2} \phi_{3}$, and $\phi_{4}$ are the price of four corresponding parts.

To get maximum utility we have to optimize the value of $\mathrm{k}$ .The value of or number of secondary users taking part in sensing depends on application in we are using it if the energy is of greater importance the value of $\mathrm{k}$ will be less because as number of cooperating secondary users increases so , does the sensing cost which degrades energy efficiency on the other hand when spectrum efficiency is of greater importance the number of cooperating secondary users increases because more the number of cooperating secondary users more efficient will be the spectrum sensing which will avoid interference to primary user. But as the number of cooperating secondary users increased further spectrum efficiency decreases because the data transmission time decreases. So, we need to optimize the number of cooperating secondary users. The maximum utility can be given by [17]

$$
\begin{gathered}
U_{2, \max }=\sum_{i=0}^{\theta}\left(\begin{array}{c}
N \\
i
\end{array}\right)\left[\mathrm{p}\left(H_{0}\right) \mathrm{p}_{\mathrm{f}}^{i}\left(1-\mathrm{p}_{\mathrm{f}}\right)^{N-i} \frac{T-t_{s}-N t_{r}}{T} \phi_{1}-\right. \\
\left.\left.\mathrm{p}\left(H_{1}\right) \mathrm{p}_{\mathrm{d}}^{i}\left(1-\mathrm{p}_{\mathrm{d}}\right)^{N-i} \frac{T-t_{s}-N t_{r}}{T} \phi_{2}\right]\right]-\frac{N t_{s}}{T} \phi_{3}-\frac{N t_{r}}{T} \phi_{4}
\end{gathered}
$$


Then optimum number of cooperating secondary users can be given by [17].

$$
\mathrm{N}_{\mathrm{opt}}=\arg _{N} \operatorname{maxU} 2_{\text {, max }}
$$

\section{SIMULATION RESULTS AND DISCUSSION}

The computer simulation is required to find out the usefulness of proposed cognitive radio frame structure and spectrum sensing schemes. So, the simulations have been performed and results have been stored. The primary user's signal is BPSK modulated signal and it is also assumed that the secondary user only transmits when primary user is absent, there is no collision among the secondary user, and all secondary users have same sensing capability and threshold of energy detection. The values of all the parameters are given below in the table, only these values will be considered in simulation if not mentioned otherwise

\subsection{Simulation parameters}

Table I. List of parameters used

\begin{tabular}{|l|l|c|c|}
\hline Sr.no. & $\begin{array}{l}\text { Name of the } \\
\text { parameter }\end{array}$ & $\begin{array}{l}\text { Vambol of } \\
\text { the } \\
\text { parameter }\end{array}$ \\
\hline 1 & $\begin{array}{l}\text { Bandwidth(primary } \\
\text { user signal) }\end{array}$ & - & - $10 \mathrm{MH}_{\mathrm{z}}$ \\
\hline 2 & $\begin{array}{l}\text { Sampling } \\
\text { frequency }\end{array}$ & $f_{s}$ & $10 \mathrm{MH}_{\mathrm{z}}$ \\
\hline 3 & Frame duration & $\mathrm{T}$ & $20 \mathrm{~ms}$ \\
\hline 4 & Sensing time & $t_{s}$ & $5 \mathrm{~ms}$ \\
\hline 5 & Reporting time & $t_{r}$ & $0.1 \mathrm{~ms}$ \\
\hline 6 & Variance of noise & $\sigma$ & 1 \\
\hline 7 & $\begin{array}{l}\text { Probability that the } \\
\text { primary user is } \\
\text { absent }\end{array}$ & $\mathrm{P}\left(\mathrm{H}_{0}\right)$ & 0.5 \\
\hline 8 & $\begin{array}{l}\text { Probability that the } \\
\text { primary user is } \\
\text { present }\end{array}$ & $\mathrm{P}\left(\mathrm{H}_{1}\right)$ & 0.5 \\
\hline
\end{tabular}

\subsection{Results and discussion}

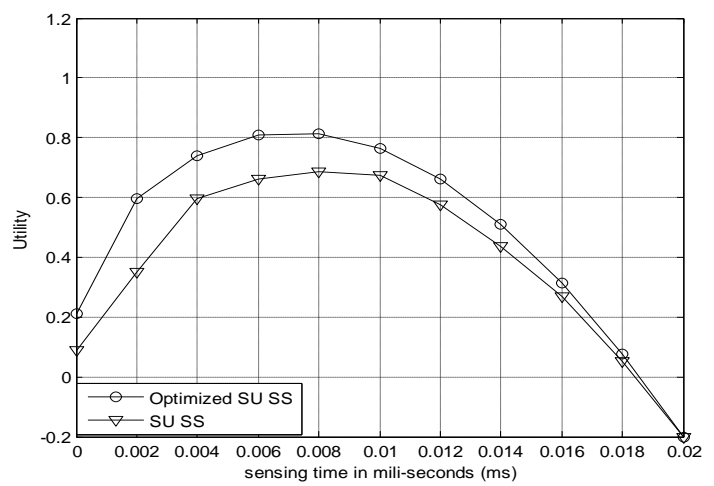

Fig.6 The utility $U_{1}$ versus sensing time $t_{s}$ graph in noncooperative single user sensing; $\phi_{1}=6 ; \phi_{2}=6 ; \phi_{3}=0.2$

Fig.6 shows $\mathrm{U}_{1}$ versus sensing time $\mathrm{t}_{\mathrm{s}}$ graph. The $\mathrm{SNR}$ of primary user's signal received at secondary user is $\gamma=$ $-10 \mathrm{~dB}$. From the graph it is quite clear that for single user spectrum sensing with the increase in sensing time utility increases because it results in greater sensing efficiency. When we further increase the sensing time the utility starts to decrease, the reason for this is the decrease in the data transmission time and the increase in the energy consumption. The other thing we can see from the graph is that the utility is greater for optimized single user spectrum sensing.

Fig.7. shows the utility $U_{1}$ versus sampling frequency $f_{s}$ graph .we have assumed that the sampling frequency is equal to the primary user's signal bandwidth, and $\phi_{3}$ which is the cost of energy consumed by secondary users for sensing and it is the function of sampling frequency. From the graph one can clearly see that when we start increasing sampling frequency the utility function increases because it improves sensing efficiency ,but when we increase it further the utility starts decreasing ; because the cost of energy consumed by secondary users for sensing increases. The other thing we can see from the graph is that the utility is greater for optimized single user spectrum sensing.

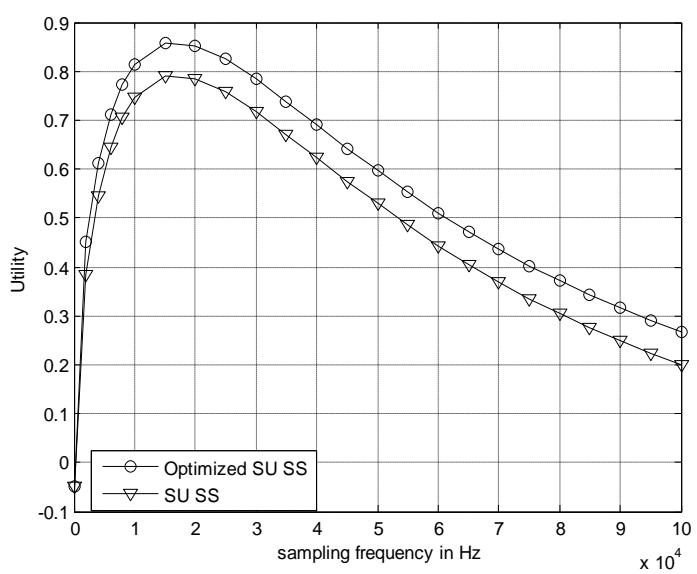

Fig.7.Shows utility $U_{1}$ versus sampling frequency $f_{s}$ graph in non-cooperative single user sensing; $\phi_{1}=6 ; \phi_{2}=6 ; \phi_{3}=$ 0.2

Fig.8 shows utility $\mathrm{U}_{1}$ versus $\mathrm{SNR}(\gamma)$ graph. The optimal $\mathrm{k}$ fusion rule is used to take the final decision. It is quite clear from the graph that the utility remains almost unchanged initially for the increase in SNR from $-18 \mathrm{~dB}$ to $-12 \mathrm{~dB}$, but when we increase the SNR further utility starts increasing and it becomes constant if we increase the SNR further after $-4 \mathrm{~dB}$. The other thing we can see from the graph is that the utility is greater for optimized single user spectrum sensing.

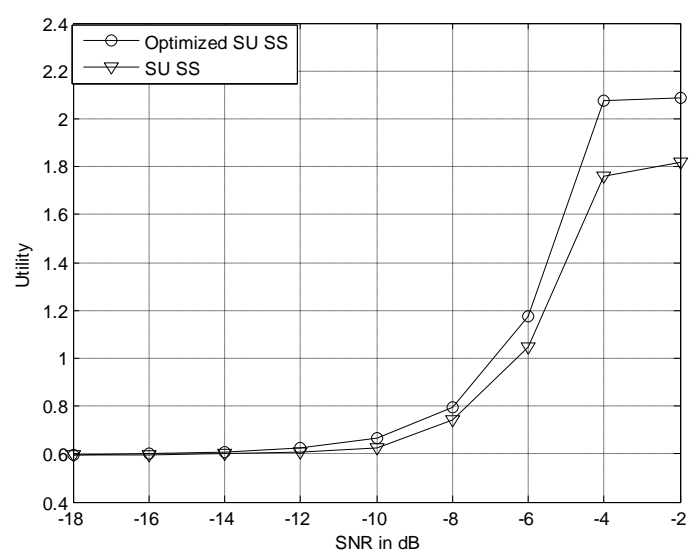

Fig.8 Shows utility $U_{q}$ vs SNR $(\gamma) \operatorname{graph} \phi_{1}=6 ; \phi_{2}=$ 4; $\phi_{3}=0.2$ 
Fig.9 shows the utility $U_{2}$ versus sampling frequency $f_{s}$ graph .we have assumed that the sampling frequency is equal to the primary user's signal bandwidth, and $\phi_{3}$ which is the cost of energy consumed by secondary users for sensing and it is the function of sampling frequency. From the graph one can clearly see that when we start increasing sampling frequency the utility function increases because it improves sensing efficiency ,but when we increase it further the utility starts decreasing ; because the cost of energy consumed by secondary users for sensing increases. The other thing we can see from the graph is that the utility is greater for optimized MSS cooperative spectrum sensing

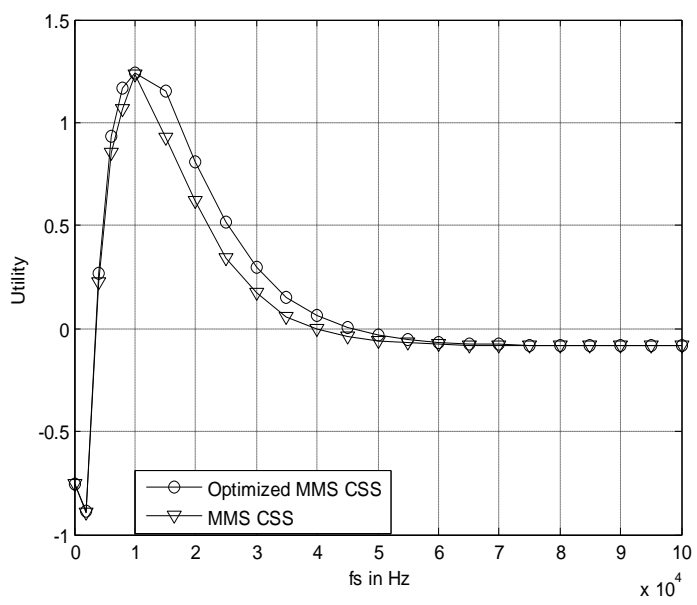

Fig.9 Shows utility $U_{2}$ versus sampling frequency $f_{s}$ graph in non-cooperative single user sensing $\phi_{1}=8 ; \phi_{2}=$ 6; $\phi_{3}=0.2 ; \emptyset_{4}=0.1 ; \mathrm{N}=15$

The optimal $\mathrm{k}$ fusion rule is used for taking the final decision. Fig.10 shows utility $\mathrm{U}_{2}$ versus $\mathrm{SNR}(\gamma)$ graph. It is quite clear from the graph that the utility remains almost unchanged initially from $-18 \mathrm{~dB}$ to $-16 \mathrm{~dB}$ SNR, but when we increase the SNR further from $-16 \mathrm{~dB}$ to $-10 \mathrm{~dB}$ utility starts increasing, because the detection of primary user's signal becomes easy. It becomes constant if we increase the SNR further. The other thing we can see from the graph is that the utility is greater for optimized single user spectrum sensing.

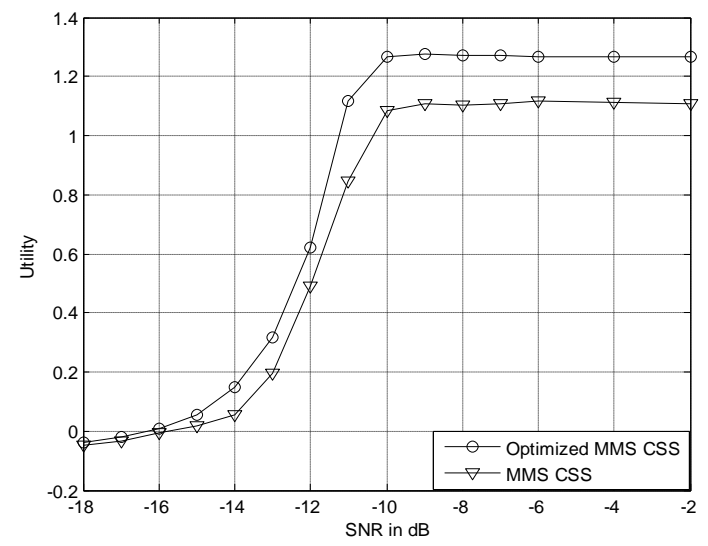

Fig.10 Shows utility $\mathbf{U}_{2}$ versus SNR $(\gamma)$ graph $\phi_{1}=$ 6; $\phi_{2}=4 ; \phi_{3}=0.2 ; \emptyset_{4}=0.1 ; N=15$

\section{CONCLUSIONS AND FUTURE SCOPE}

\subsection{Conclusions}

The proposed a time division frame structure for cognitive radio maximizes its utility for both non cooperative single user sensing and cooperative spectrum sensing. It is found that new multi-minislots cooperative spectrum sensing scheme provides time diversity gain in sensing performance. The use of fusion rule with optimal decision threshold results in greater spectrum and energy efficiency in spectrum sensing , and there is an optimal number of cooperative sensing users one have to find to maximize utility because as the number of cooperative spectrum sensing users increases ,the energy consumed by them increases, so ,does the sensing cost which degrades energy efficiency.

\subsection{Future scope}

In this paper fixed values of costs used, if optimized values of costs will be used the utility will be higher because optimized values of costs will be less than the fixed values of costs used in this paper

\section{ACKNOWLEDGEMENT}

We are very thankful to the M.Tech and Ph.D. scholars of department of ECE M.A.N.I.T. for their valuable inputs.

\section{REFERENCES}

[1] FCC, ET Docket No 03-222 Notice of proposed rulemaking and order, December 2003

[2] Ian F. Akyildiz, Won-Yeol Lee, Mehmet C. Vuran *, Shantidev Mohanty, "Next generation/dynamic spectrum access/cognitive radio wireless networks: A survey," Computer Networks 50 (2006) 2127-2159.

[3] Suzan Bayhan, Salim Eryigit, Fatih Alag“oz, and Tuna Tugcu 'Low Complexity Uplink Schedulers for EnergyEfficient Cognitive Radio Networks," IEEE wireless communications e letters, vol. 2, no. 3, June 2013

[4] Hang Su and Xi Zhang"Energy-Efficient Spectrum Sensing for Cognitive Radio Networks,' IEEE ICC , 2010

[5] Stergios Stotas, and Arumugam Nallanathan "On the Throughput and Spectrum Sensing Enhancement of Opportunistic Spectrum Access Cognitive Radio Networks,"IEEE Trans. On Wireless Communications, vol. 11, no. 1, January 2012

[6] Wenshan Yin, Pinyi Ren, , Qinghe Du, and Yichen Wang"Delay and Throughput Oriented Continuous Spectrum Sensing Schemes in Cognitive Radio Networks," IEEE transactions on wireless communications, vol. 11, no. 6, June 2012

[7] Thanayankizil L., Kailas, A., (2008), "Spectrum Sensing Techniques (II): Receiver Detection and Interference Management,, http://aravind.kailas. googlepages.com

[8] D.D.Ariananda, M.K.Lakshmanan, H.Nikookar , “A Survey on Spectrum Sensing Techniques for Cognitive Radio", Wireless VITAE'09, Aalborg, Denmark, pp: 74 $79,2009$.

[9] Sun C, Zhang W, Letaief KB. "Cooperative spectrum sensing for cognitive radios under bandwidth constraints," In: Proc. of IEEE Wireless Communications and Networking Conference. 2007. p. 27-32. 
[10] Hang Hu, Hang Zhang*, Hong Yu, and Yi Chen "Spectrum-energy-efficient sensing with novel frame structure in cognitive radio networks," Int. J. Electron. Commun. (AEÜ) 68 (2014) 1065-1072.

[11] Tevfik Yucek and Huseyin Arslan "A survey of spectrum sensing algorithms for cognitive radio applications," IEEE communications surveys and tutorials .vol.11. no.1, first quarter 2009

[12] Yu H, Tang W, Li S."Optimization of cooperative spectrum sensing with sensing user selection in cognitive radio networks," EURASIP Journal on Wireless Communications and Networking, 2011;2011(208):1-8.

[13] Liang YC, Zeng Y, Peh ECY, Hoang AT. Sensingthroughput tradeoff for cognitive radio networks. IEEE Transactions on Wireless communications 2008;7(4):1326-37.

[14] Mrs. R. S. Kale (Sandikar), Dr. Vijay M. Wadhai, and Dr. Jagdish B. Helonde" Efficient spectrum sensing in cognitive radio using energy detection method with new threshold formulation," International Conference on Microelectronics, Communication and Renewable Energy, 2013

[15] Z. Chair and P. K. Varshney, "Optimal data fusion in multiple sensor detection systems," IEEE Trans. Aerospace Elect. Syst., vol. 22, no. 1, pp. 98-101, Jan. 1986

[16] Ying-Chang Liang, Yonghong Zeng, Edward C.Y. Peh, and Anh Tuan Hoang, "Sensing throughput trade-off for cognitive radio networks," IEEE Transaction on wireless communications vol.7, NO.4, April 2008.

[17] Hang Hu, Hang Zhang, and Yewen Guan"Cost-Efficient Cooperative Spectrum Sensing via Utility Maximization," Sixth International Conference on Wireless Communications and Signal,2014. 